\title{
Efficient progressive failure analysis of multi-stringer stiffened composite panels through a two-way loose coupling global-local approach
}

\author{
Margarita Akterskaia*, Eelco Jansen, Sina Hühne, Raimund Rolfes \\ Institute of Structural Analysis, Leibniz Universität Hannover, Appelstr. 9A, 30167 \\ Hannover, Germany
}

\begin{abstract}
A two-way coupling global-local finite element approach, which has demonstrated its potential on the basis of representative test cases in earlier work, is used for the progressive failure analysis of large stiffened composite panels. In order to realize the capability of the approach to analyze larger panels, the efficiency of the analysis is enhanced, and improved rules for the choice of the size of local models are developed.

The potential to carry out a progressive failure analysis for large stiffened panels is illustrated firstly through the analysis of a two-stringer panel with a local defect, in which the adjusted rules to define the local models are applied, and subsequently concretized by applying the approach to a large stiffened composite panel with five stringers. A comparison between the results of the global-local coupling analysis and the shell element reference analysis are demonstrated and the results are discussed. The results of the numerical analyses of the large panel are also compared with experimental results available.
\end{abstract}

Keywords: Composite structures, Stiffened panels, Progressive failure analysis, Multiscale analysis, Global-local method, Postbuckling

\footnotetext{
* Corresponding author

Email address: m.akterskaia@isd.uni-hannover.de (Margarita Akterskaia)
} 


\section{Introduction}

Due to their favorable strength over weight ratio and stiffness over weight ratio, fiber-reinforced composites are often used in aircraft applications. For post-buckled designs, an efficient analysis capability for the nonlinear finite ele5 ment analysis of panel-type structures is required. For design scenarios, in which material degradation can occur before the failure load is reached, a capability of a progressive failure analysis should be available, in order to predict the failure load of the structure with sufficient accuracy. Two-way loose coupling methods provide a possibility to simulate the post-buckling progressive failure behavior coupling global-local finite element approach, which has demonstrated its potential on the basis of representative test cases in earlier work, is used for the progressive failure analysis of large, multi-stringer stiffened composite panels.

\subsection{Progressive failure of multi-stringer stiffened panels}

As stiffened composite panels are widely applied in aircraft design, they are used as model cases in the present paper. Stiffened panels usually consist of a straight or curved plate, called skin, strengthened by stiffeners, also referred to as stringers. Compression load which is one of the most common loads for this kind of panels is considered in this paper. However, the loose-coupling method is not limited by this type of load. To describe structural behavior of the thin stiffened panel under compression it is useful to remember that usually linear-elasticity is followed by initial buckling [1. In that case, only the skin is expected to buckle, while the stringers do not show any deformation. The overall stiffness of the panel is reduced, but the structure is still capable of withstanding increased loads. When the compression load is increased, stringers eventually also start to buckle until the ultimate load of total failure. This can be noticed by a sudden drop of load-displacement curve.

To date, the experiments on postbuckling behavior of curved and flat panels with several stringers have been performed by several authors [2, 3, 4, 5, 6]. 
Imperfections, such as holes and debonding between skin and stringer, were also considered. Degenhardt et al. [2] described the testing of a stiffened panel and an unstiffened cylinder. In the work of Orifici et al. [3], multi-stringers composite panels were tested experimentally, and analyzed numerically under compressive loading. The panels demonstrated matrix and fiber damage as well

${ }_{35}$ as delaminations between skin and stiffener and in the stiffener blade area before the ultimate failure occurred. Lauterbach et al. [4] experimentally tested intact and predamaged five-stringer curved CFRP (carbon fiber reinforced polymer) panels with compressive axial load until final collapse. Numerical analysis took into account delamination, matrix, fiber cracking and matrix-fiber shear failure with following reduction of material properties, though discrepancies between experimental and numerical results were observed, especially in the stiffness evaluation and total failure load. In another study conducted by Zimmermann et al. [5], experimental data on the buckling and postbuckling behavior of stiffened curved composite panels were generated. Panels with even and odd number of stiffeners, different ratios of curvature and different skin thickness were examined. Wagner and Balzani [6] applied shell elements to model a fivestringer laminated panel, by using a brittle material degradation model and comparing it to experimental results. Matrix, fiber and matrix-fiber debonding were considered as damage mechanisms, and the extended Hashin criteria were utilized.

\subsection{Coupling methods}

In the last few decades, coupling methods have attracted attention because of their efficiency meeting the needs of progressive damage analysis. Global-local coupling analyses are based on the following idea. On the one hand, at the global

level sufficiently accurate results are obtained at relatively low computational cost. On the other hand, analysis at the local level, with smaller area of interest, could regard in detail geometrical and material nonlinearities usually ignored during the global analysis.

The submodeling technique or so-called zooming technique was successfully 
${ }_{60}$ applied in the past for one-way global-local transition in order to obtain accurate results at the local level. Examples can be found in the works of Mote [7], Noor [8], Mao and Sun [9], and Whitcomb [10]. In contrast, during a twoway coupling, the information is exchanged in both directions, accounting for interactions of global and local effects. To cite a few of the recent global-

${ }_{65}$ local approaches, the multiscale projection method by Löhnert and Belytischko [11] and the adaptive progressive damage modeling technique by Labeas et al. [12] are to mentioned. Two-way loose coupling methods [13, 14, 15] provide a promising way to analyze various types of nonlinear structural analysis problems in an efficient way.

The global-local method proposed by Hühne [15] has been applied in the context of postbuckling problems of composite structures with damage and makes use of an efficient homogenization technique for the degraded material properties that are transferred from the local to global levels.

In the approach proposed by Hühne et al. [15], the post-buckling behavior is ${ }_{75}$ analyzed through an analysis with a global model discretized with shell elements and the areas in which damage can be expected to occur are identified. In order to obtain these critical damaged areas, a global analysis is performed. The critical regions are examined during the next step - local analysis with solid elements and a finer mesh element discretization. Displacements from the 80 global model are transferred to the selected boundary nodes of the local model by means of Abaqus shell-to-solid submodeling [16]. The user material model by Linde et al. [17] is used to define the material degradation. After calculation of the degraded properties, so-called local part tests are carried out layerwise, in order to obtain equivalent properties for each global element and to transfer

${ }_{85}$ them back to the global model. With the new global degraded properties, a restart of the global analysis from the last critical state is carried out. These coupling steps are repeated so long as convergence in reaction forces in the direction of applied load is not obtained. In our case, the reaction force from global analysis is also compared to the reaction force of the reference solid model. 
The two-way coupling global-local finite element has demonstrated its potential on the basis of representative test cases, in particular one-stringer panel [15. In the present work, the approach is used for the progressive failure analysis of large stiffened composite panels. In order to realize the capability of the

95 approach to analyze larger panels, the efficiency of the analysis is enhanced, and improved rules for the choice of the size of local models are developed. The potential to carry out a progressive failure analysis for large stiffened panels will be demonstrated in a first step through the analysis of a two-stringer panel with a local defect, and furthermore by analyzing a large stiffened composite panel with five stringers.

\section{Enhancements of the two-way coupling approach for multi-stringer panels}

As a necessary prerequisite for the efficient analysis of large panels using the loose coupling global-local approach presented by by Hühne [15, the appropriate definition of the local models within this approach is addressed in the following. The objective of this section is to formulate a guideline for the creation of local models for thin stiffened panels, thereby proposing a modification of the approach used in earlier work. The typical academic one-stringer panel test case is analyzed to check the appropriateness of the new formulation. In addition, a study on the mesh convergence in the local areas is conducted in order to check the influence of the mesh element size refinement on the damage evolution.

\subsection{Local model definition}

The submodeling technique is used to investigate the detailed behavior of a particular region of the structure [18]. In order to define the regions which should be included in the local model, in the following the identification of these areas of interest is addressed, requiring an understanding of the behavior of the structural parts adjacent to the structural element, in which damage is detected. 

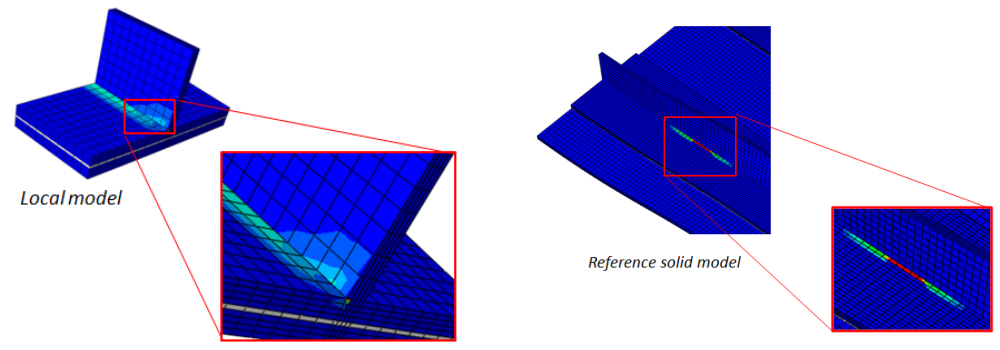

Figure 1: Matrix damage in the local model (left) and in the solid reference model (right).

For stiffened panels under compression, after each global analysis, all parts of the panel are checked for damage. This check eliminates the risk of missing the failure initiation in a particular area. Therefore, there is no need to consider the three structural elements skin, stringer foot and stringer web all together in one local model, if, for example, only the stringer foot is damaged at the global level. This important assumption should decrease the size of the local model and become a new guideline for the correct definition of local model geometry.

Moreover, Fig. 1 demonstrates an issue arising from the incorrect definition of a local model. Damage can be observed in the stringer area of the local model. The absence of damage in the reference solid element model at the same load level indicates that this damage in the local model is artificial and due to edge effects. One should therefore try to avoid stress concentrations due to the boundaries of the local and pay additional attention to stress convergence [19]. This issue is addressed in the following.

The spurious stress concentrations at the edges of local models can lead to inaccurate results. This might considerably affect the evaluation of the homogenized properties which are transferred back to the global level. A first possibility ${ }_{135}$ is to surround the local models by material which in the coupling analysis is assumed not to degrade, in order to add an additional local layer of elements at each edge where the boundary conditions are applied. Another option is to increase the local model, thereby involving the nearest global elements. The second approach is preferred due to the submodeling technique. The reason is 
be interpolated only to the corresponding driven nodes of the local model lying within a certain tolerance [16]. To satisfy the submodeling procedure and to obtain an accurate interpolation of the displacements at the boundaries, the local nodes should lie in the vicinity of the global nodes.

145 during a global analysis in the following way. If a few elements in the foot of the stiffened panel are damaged, then not only these elements, but also the corresponding adjacent skin and stiffener elements will be included in the local model. Based on a plane stress assumption as well as on the idea that loss there is no need to examine the stiffener until the moment that the failure is predicted through the global analysis. As the whole global-local approach is iterative, there is no risk in missing damage evolution in the adjacent stringer elements. There is further no need to include the adjacent skin elements in the local model until damage has been detected in these skin elements. The new local model rules proposed require surrounding the damaged area by at least one neighboring global element. Further, only those parts of the model corresponding to the specific structural element in which damage was identified should be considered, provided that no stress concentrations are expected at the connection of these structural elements.

Summarizing, on the one hand, the size of the local model will be increased in-plane by adding elements corresponding to the surrounding global elements. On the other hand, the size of the local model will be decreased in the case of damage in the stringer foot by not considering adjacent skin and stringer web parts until in those parts damage has been detected at the global level. This approach will be used for the stiffened panels in this paper in the cases in which failure initiates in the stringer foot. These rules can be extended to other types of composite structures and load cases. By considering the load distribution and redistribution expected in the structure after initial damage, one can identify 
adjacent areas that are not expected to be influenced in the same local model analysis step.

\subsection{Analysis of one-stringer composite panel with new local model rules}

In order to evaluate their effect on the failure growth prediction, the new local model rules discussed in the previous section are applied in a progressive failure analysis using the global-local coupling approach for a one-stringer panel. Reference models with shell and solid elements have already been analyzed in the framework of earlier work on an academic one-stringer panel [15]. Geometry as well as material linear properties for the global models are the same as in these previous analyses. In the present case, the new rules to define local models are employed. Prescribed displacements for the progressive analysis and subsequent coupling loops are applied similarly to the earlier analysis in four steps, in order to ensure consistency when comparing results with the results obtained earlier [15]: prescribed displacements of $0.56 \mathrm{~mm}, 0.60 \mathrm{~mm}, 0.63 \mathrm{~mm}$ and $0.67 \mathrm{~mm}$, respectively. So-called "overlay" plots of the separated global shell model and the local solid models for these four coupling loops are presented in Fig. 2. The relative error of total failure predicted by global-local analysis with the new local models is $0.65 \%$ as compared with the reference solid model, which is satisfactory and comparable with the relative error obtained with the local models used in the earlier analysis $(0.53 \%)$. Not only the number of global elements examined during the local step has been decreased, also the number of local part tests has been decreased, as they are performed only for damaged global elements. The properties of non-damaged global edge elements in local models are not homogenized, because this can lead to spurious results.

The damage evolution detected via global-local analysis with the new local model rules is similar to the one in observed in the previous global-local analysis [15. Matrix cracking in the foot is first detected, see Fig. 3 and progresses with increasing compressive load. Next, fiber damage is found in the foot, followed by the global failure of the panel.

The corresponding load-displacement curve is plotted in Fig. 4, showing 


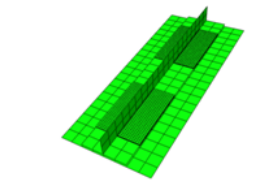

Loop 1: $\mathrm{u}=0.56 \mathrm{~mm}$

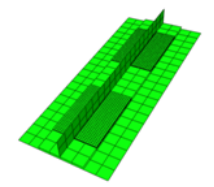

Loop 2: $\mathrm{u}=0.60 \mathrm{~mm}$

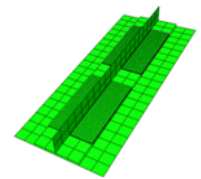

Loop 3: $\mathrm{u}=0.63 \mathrm{~mm}$

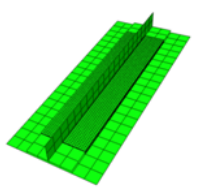

Loop 4: $\mathrm{u}=0.67 \mathrm{~mm}$

Figure 2: Overlay plots for global and local models from coupling loops 1-4.

a Coupling loop 1, global model: matrix damage
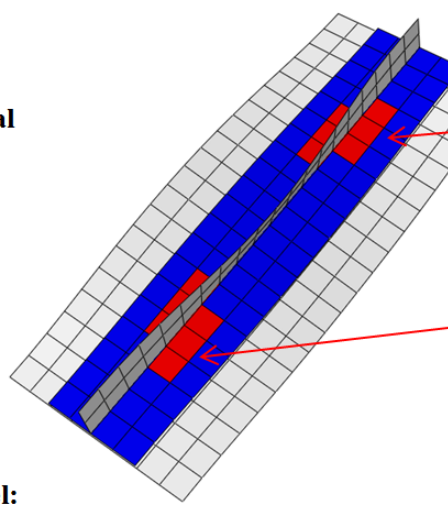

b
Region 1, local model: matrix damage

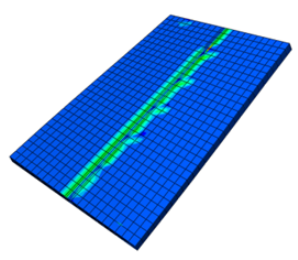

Region 2

Region 1 Region 2, local model:
matrix damage

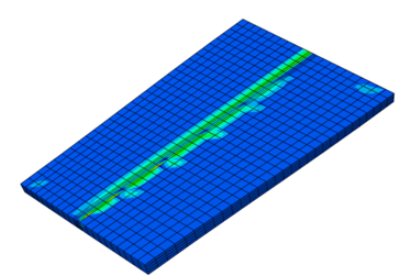

Figure 3: Coupling loop 1. (a) First critical global areas related to matrix failure, (b) matrix damage initiation for the Regions 1 and 2 on the local level.

that the global-local coupling model results are similar to the load-displacement path of a reference model with solid elements, while initial buckling of the structure occurs slightly earlier. The response curves for the reference solid element model with solid elements and for the coupling analysis after initial buckling also show the same stiffness in the postbuckling region. The first failure in both analyses occurs in the matrix of the stringer foot in the postbuckling regime. Global failure is obtained in the reference solid element model after 


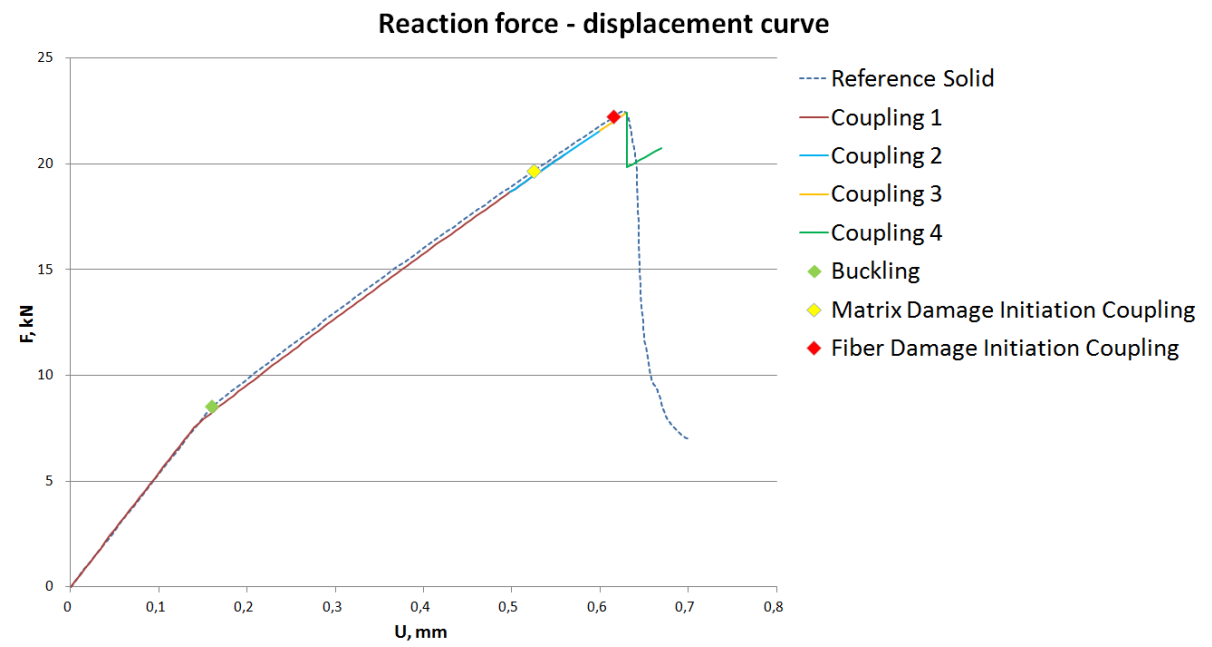

Figure 4: Load-displacement curves of the coupling and reference analyses.

the occurrence of fiber damage and is satisfactorily captured by the global-local coupling procedure. not only lead to more accurate stress and displacement fields, but also to a different damage evolution. This last aspect also needs to be addressed, proving the reliability of the coupling method used. 
A coupling analysis is performed for a test case of a one-stringer panel. The local areas are detected and defined using the Linde damage criterion and they are modeled with twice as many elements in each in-plane direction as in the analysis, described in the previous section, with the result that the number of elements is quadrupled. The new local model rules from the section 2.1 are again applied. For consistency, a reference solid element model was created with the same size of elements as used at the refined local level. The difference between the global failure load level predicted by the global-local coupling method and the reference solid element analyses is $0.19 \%$, while the difference with the coarser local models is $0.65 \%$. The relatively small increase in accuracy obtained using the finer discretization demonstrates that the results in this case do not significantly depend on the mesh element size. Load-displacement curves for the reference solid and coupling analyses are presented in Fig. 5 .

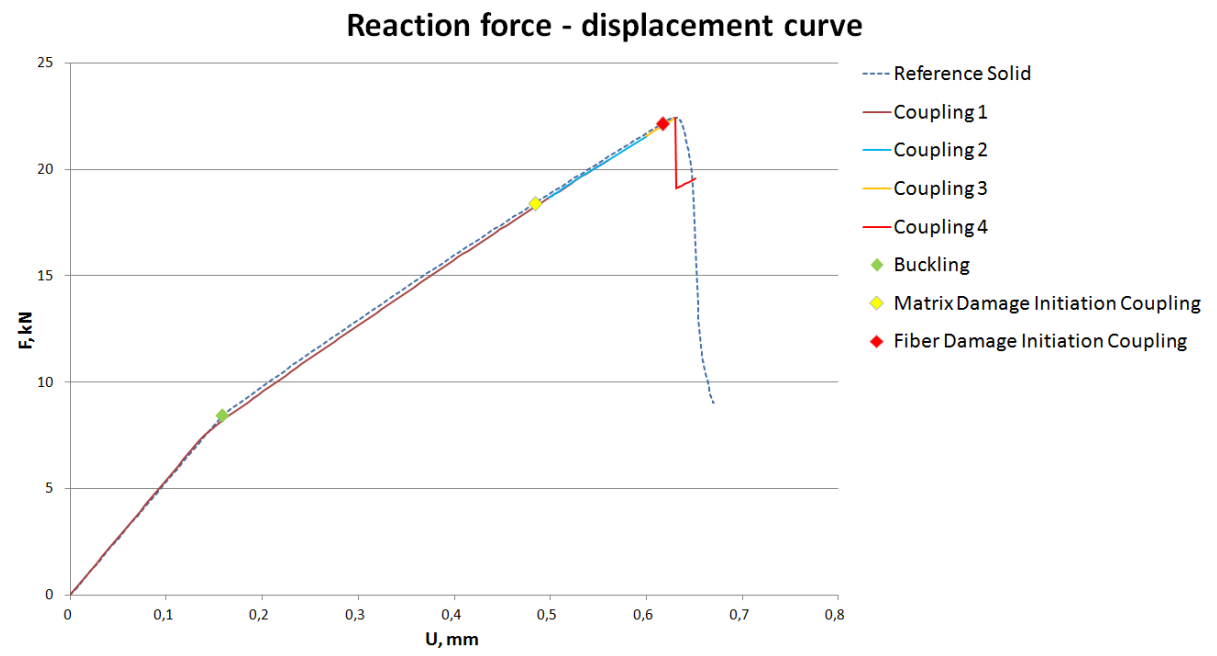

Figure 5: Load-displacement curves of the coupling and reference analysis. Verifying mesh dependency.

\section{Two-stringer composite panel with local defect}

In this section, as a first step towards the analysis of large panels, a stiffened panel with two stringers is analyzed by means of the two-way coupling method 
including the enhancements presented in Section 2

Results obtained by Hühne [15] for one-stringer panels show a reasonably good agreement with the corresponding reference solid element models. Due to the panel configuration and loading conditions, the one-stringer panel showed damage in large areas of the structure. In the present case of the two-stringer panel, the damage is expected to grow in a localized area. The analysis of local initial defect, for instance due a flaw in the manufacturing process, also has a practical significance, e.g. [20].

\subsection{Model description}

250

The application of the refined method is demonstrated on a flat stiffened panel with two T-stringers shown in Fig. 6, see Table 1 and 2 for geometrical and material characteristics respectively. The debonding as an initial localized defect was incorporated between skin and a flange of the stringer where $\mathrm{d}=$ $25 \mathrm{~mm}$, and the distance between clamped edge and debonded area was $l_{d}=$ $15 \mathrm{~mm}$. The unidirectional layers are of $0.25 \mathrm{~mm}$ thickness with a symmetric composite layup $\left[0^{\circ}, 90^{\circ}\right]_{s}$.

Progressive damage was examined under the following boundary conditions. The fully clamped transverse edge and the opposite transverse edge constrained in all directions except the longitudinal direction, where compressive load was imposed as displacement. The longitudinal edges are free. The debonding area is assumed not to grow under the load applied.

\subsection{Coupling results}

Four coupling loops with consequently increased displacements were performed to detect the final damage. With an axial compressive displacement of $\mathrm{u}=0.37 \mathrm{~mm}$, matrix failure was observed in the debonded area, according to the Linde criterion [17. Increase of the displacement to $\mathrm{u}=0.41 \mathrm{~mm}$ predicted a matrix damage initiation in the skin between two stringers. Damage spreads in the debonded region in the foot part of the panel with a displacement of 
Table 1: Geometry of a two-stringer composite panel.

\begin{tabular}{lll}
\hline Description & Symbol & Value $(\mathbf{m m})$ \\
\hline Panel length & $\mathrm{l}$ & 100 \\
Panel width & $\mathrm{w}$ & 80 \\
Stringer width & $w_{\text {stringer }}$ & 20 \\
Stringer height & $\mathrm{h}$ & 8 \\
Laminate thickness & $\mathrm{t}$ & 1 \\
Adhesive thickness & $t_{\text {glue }}$ & 0.2 \\
\hline
\end{tabular}

Table 2: Material data for composite and adhesive.

\begin{tabular}{llll}
\hline Stiffness properties & Value & Strength and fracture properties & Value \\
\hline Young's modulus in 1-direction $E_{11}$ & $146.5 \mathrm{GPa}$ & Tensile strength in 1-direction $X_{T}$ & $2.583 \mathrm{GPa}$ \\
Young's modulus in 2-direction $E_{22}$ & $9.7 \mathrm{GPa}$ & Compressive strength in 1-direction $X_{C}$ & $1.483 \mathrm{GPa}$ \\
Shear modulus in 12-plane $G_{12}$ & $5.1 \mathrm{GPa}$ & Tensile strength in 1-direction $Y_{T}$ & $0.092 \mathrm{GPa}$ \\
Poisson's ratio $\nu_{12}$ & 0.28 & Compressive strength in 2-direction $Y_{C}$ & $0.270 \mathrm{GPa}$ \\
Young's modulus of adhesive $E_{\text {glue }}$ & $3.0 \mathrm{GPa}$ & Shear strength in 12-plane $S_{A}$ & $0.106 \mathrm{GPa}$ \\
Poisson's ratio of adhesive $\nu_{\text {glue }}$ & 0.4 & Fracture energy of fibre $G_{f}$ & $12.5 \mathrm{~N} / \mathrm{mm}$ \\
- & - & Fracture energy of matrix $G_{m}$ & $1.0 \mathrm{~N} / \mathrm{mm}$ \\
\hline
\end{tabular}




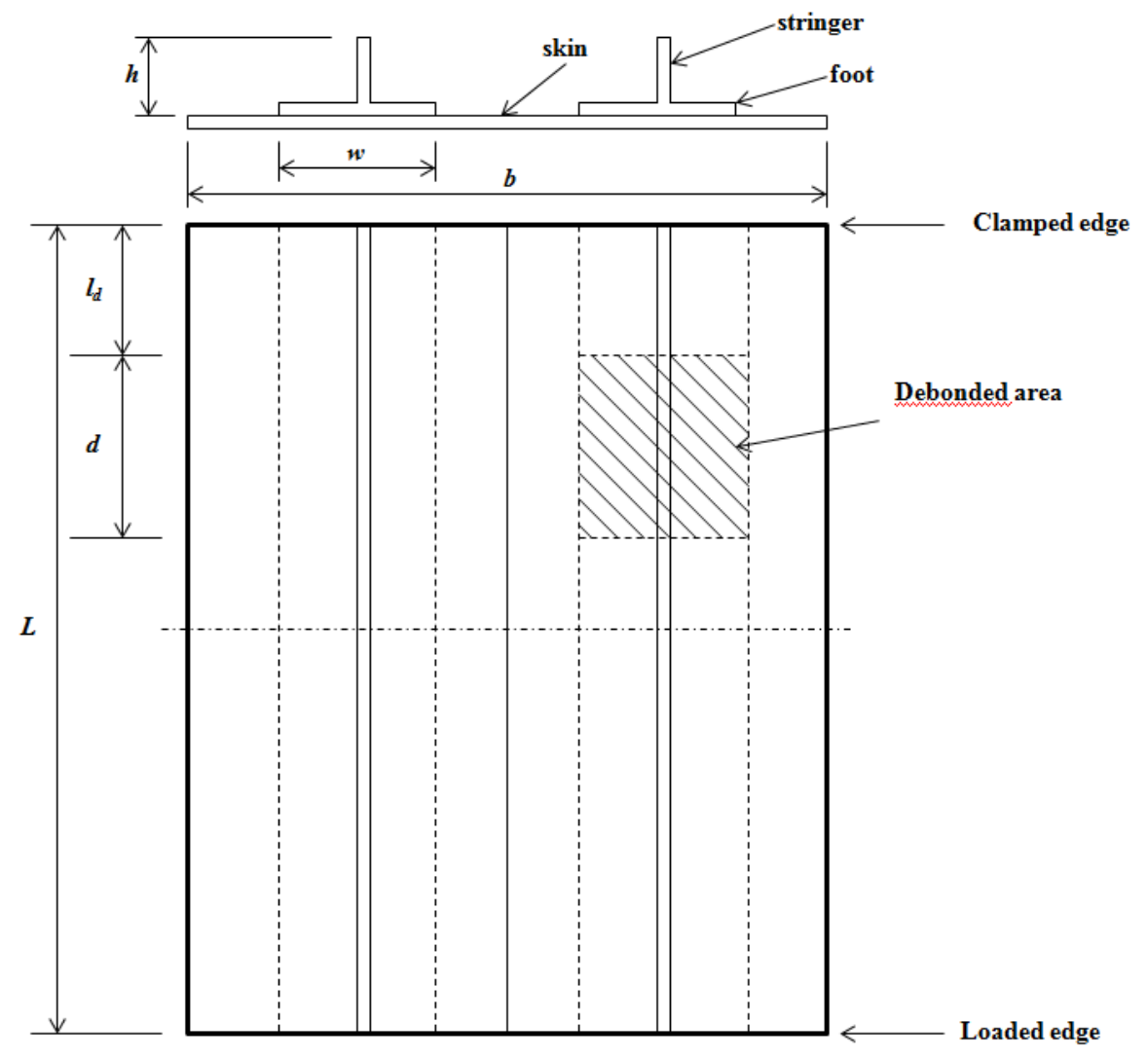

Figure 6: Geometry of a two-stringer composite panel with debonding.

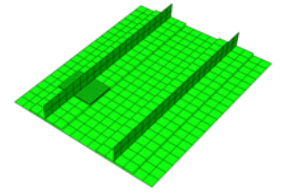

Loop 1: $\mathrm{u}=0.37 \mathrm{~mm}$

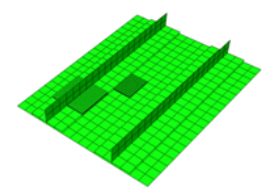

Loop 2: $\mathrm{u}=0.41 \mathrm{~mm}$

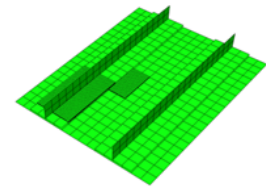

Loop 3: $\mathrm{u}=0.43 \mathrm{~mm}$

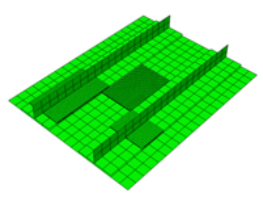

Loop 4: $\mathrm{u}=0.45 \mathrm{~mm}$

Figure 7: Overlay plots of global and local models from coupling loops 1-4. Two-stringer panel

$\mathrm{u}=0.43 \mathrm{~mm}$. Global failure occurs when fiber failure in the stringer foot and in

the skin occur. The damage evolution is shown in Fig. 7. 


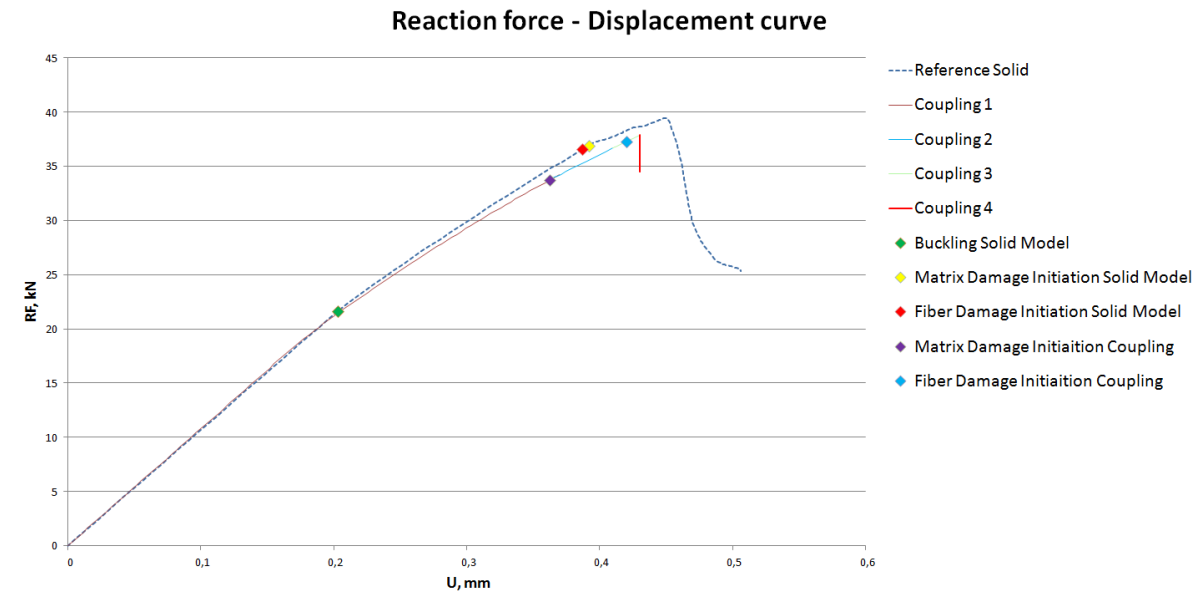

Figure 8: Load-displacement curves of coupling iterations for a two-stringer panel with initial debonding in comparison with reference results

Fig. 8 displays load-displacement curves of four iteration loops obtained from the global-local coupling procedure as compared with the reference solid element model analysis results. There is a deviation of approximately $4 \%$ between the maximum load predicted by the coupling analysis and that of the solid reference analysis. From the load-displacement curve in Fig. 8, it can be seen that the coupling analysis predicts lower maximum prescribed displacements than the reference solid element model. While the solid element reference models carried a certain amount of additional load after onset of fiber degradation, the panels examined using the coupling procedure collapsed after first fiber failure. The stiffness decrease in the last region of the postbuckling regime is slightly higher than for the reference solid element model.

For this debonded panel with localized damage growth, a reasonably good agreement with the reference solid element model analysis was demonstrated. The new local model rules also played an important role, avoiding unnecessary calculations of undamaged parts and ensuring an appropriate stress and displacement evaluation at the local level. For this case of a two-stringer panel with a local defect, the potential of capturing the localized damage evolution of a larger structure by means of the global-local coupling approach could already 
be demonstrated.

\section{Curved five-stringer composite panel}

In this section, a realistic, large composite stiffened panel with five stringers is analyzed by means of the two-way coupling method including the enhancements presented in Section 2, concretizing the potential to carry out a progressive failure analysis for large stiffened panels. Larger panels are of particular interest for the global-local techniques, as the localized failure phenomena play an important role in the failure behaviour of the panel as a whole.

\subsection{Model description}

Characteristics of a realistic five-stringer panel, that was tested experimentally in the COCOMAT project [2] denoted as P29, were used. During the tests, this panel was loaded cyclically until damage was generated. Wagner and Balzani [6] performed a numerical analysis for the panel with initial damage. In the present analysis, the panel without predamage is considered.

The panel was composed of a curved skin and five T-shaped stringers, see Fig. 9. An axial displacement was applied in compression to one of the transverse edges, while the opposite edge was fully clamped and both transverse edges were potted. The geometry parameters have been taken from [6] and are summarized in Table 3 ,

The composite laminate properties for this panel, made of unidirectional CFRC IM7/8552 plies, have been taken from the aforementioned papers. Material properties correspond to those used for the two-stringer panel, see Table 2 The symmetric composite layups for the skin and for the stringer are $\left[90^{\circ},+45^{\circ},-45^{\circ}, 0^{\circ}\right]_{s}$ and $\left[\left(+45^{\circ},-45^{\circ}\right)_{3}, 0_{6}^{\circ}\right]_{s}$ respectively [2].

\subsection{Coupling results}

A global model consisted of 17,940 shell elements, while the local models representing skin and stringer areas consisted of a maximum of 79,380 solid elements with a mesh refinement of 6 times the value used at the global level. 


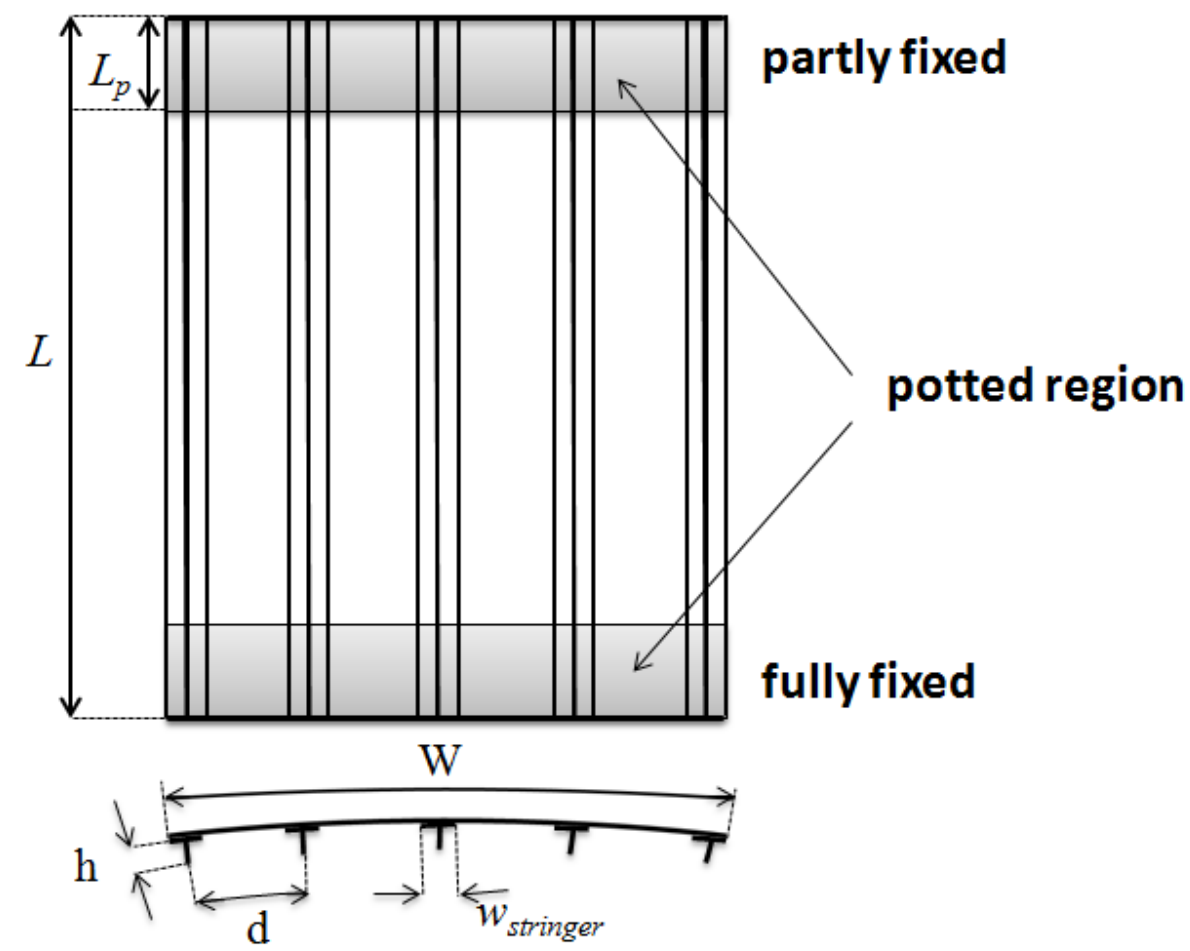

Figure 9: Curved five-stringer composite panel.

Four coupling steps are applied to simulate the progressive failure analysis in the postbuckling regime. Onset of the intralaminar failure is detected at a matrix of the skin located between stringers followed by fiber damage at the middle stringer in the next coupling step. Further damaged regions are found near the edges of the first and the fifth stringer. Final collapse of the panel occurs following spreading of fiber damage in the stringers at a prescribed displacement of $3.28 \mathrm{~mm}$.

For reference purposes, the panel with shell elements including a material degradation model based on Linde's approach was used. A numerical calculation of a reference model with solid elements and a mesh discretization corresponding to the local models would require a very high computational time and was not done for the present model. 
Table 3: Geometry of a five-stringer composite panel.

\begin{tabular}{lll}
\hline Description & Symbol & Value $(\mathbf{m m})$ \\
\hline Panel length & $\mathrm{L}$ & 780.5 \\
Potted length & $L_{p}$ & 60.25 \\
Panel width & $\mathrm{W}$ & 560.5 \\
Stringer width & $w_{\text {stringer }}$ & 32 \\
Stringer height & $\mathrm{h}$ & 14.3 \\
Skin thickness & $t_{s}$ & 0.98 \\
Stringer thickness & $t_{s}$ & 2.9 \\
Panel radious & $\mathrm{R}$ & 848 \\
\hline
\end{tabular}

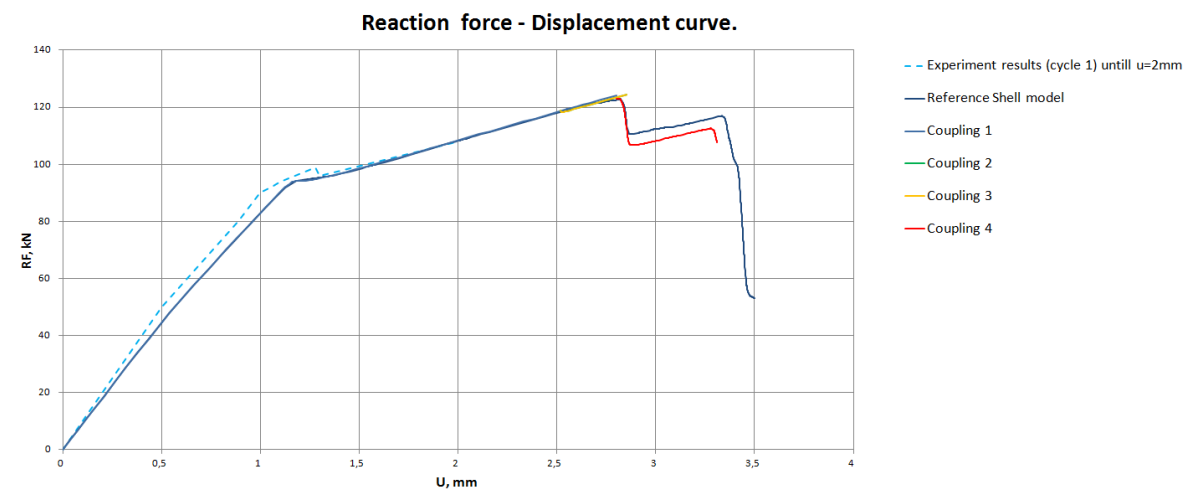

Figure 10: Load-displacement curves of a five-stringer panel. Comparison between results of global-local approach, results of reference panel analysis and experimental results.

Load-displacement curves for a reference panel with shell elements, for coupling results and for experiment results are displayed in Fig. 10. During the experiment, the panel was compressed only until the displacement of $2 \mathrm{~mm}$. The response curves are in reasonable agreement before buckling and also after first buckling has occurred. First buckling (local skin buckling) obtained in the numerical analysis corresponds to a prescibed displacement of $u=1.13 \mathrm{~mm}$. 335 The "overlay" plots showing the damaged areas are presented in Fig. 11. First 
failure occurred in the matrix of the skin between the second and third stringer at a prescribed displacement of $2.55 \mathrm{~mm}$ followed by initial fiber damage in the skin under the middle stringer at the prescribed displacement of $2.68 \mathrm{~mm}$. Subsequently, initial fiber failure was detected in the first and fifth stringer in three areas near the panel edge at a prescribed displacement of $2.69 \mathrm{~mm}$, with further damage growth in the other regions with damage. Final failure of the panel occurred following significant spreading of fiber damage at a prescribed displacement level of $3.28 \mathrm{~mm}$.

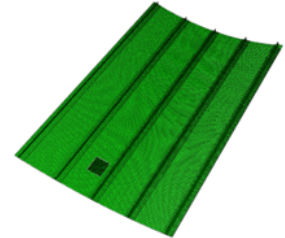

Loop $1: \mathrm{u}=2.80 \mathrm{~mm}$

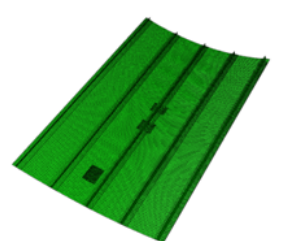

Loop 2: $\mathrm{u}=2.85 \mathrm{~mm}$

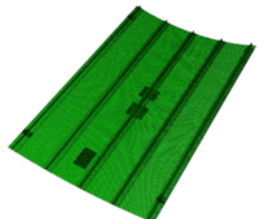

Loop 3: $\mathrm{u}=3.20 \mathrm{~mm}$

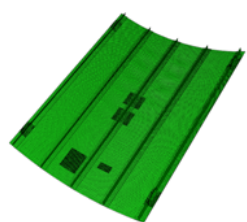

Loop $4: \mathrm{u}=3.30 \mathrm{~mm}$

Figure 11: Overlay plots of global and local models. Five-stringer model

It can be seen that the prescribed displacement of the forth coupling loop is

345 elements, the procedure can also for this large structure be carried out with a reasonable computational time.

\section{Conclusion}

A two-way coupling global-local finite element approach, which has demon- 
used for the progressive failure analysis of large multi-stringer stiffened composite panels. In order to realize the capability of the approach to analyze larger panels, the efficiency of the analysis was enhanced, and improved rules for the choice of the size of local models were developed.

As a first step towards the analysis of large panels, a case of a two-stringer panel with a local defect was analyzed using the adjusted rules to define the local models. Load-displacement curves of the coupling analysis and the solid element reference analysis were in reasonably good agreement; This holds for the maximum load levels predicted by both analyses as well as for the sequence of damage occurrence.

The potential to carry out a progressive failure analysis for large stiffened panels was concretized by analyzing a large stiffened composite panel with five stringers. A reference model with shell elements and material degradation was used for a comparison. A reasonable agreement between the results of the coupling analysis and the shell element reference analysis was demonstrated. The numerical results were also compared with experimental results available. The load-displacement response curves observed showed, despite the small difference in the prebuckling stiffness, a reasonable agreement in the postbuckling regime.

\section{Acknowledgments}

The research leading to these results has received funding from European Union's Horizon 2020 research and innovation program (FULLCOMP/20152019) under Marie Sklodowska-Curie actions grant agreement number 642121. The provided financial support is gratefully acknowledged by the authors.

\section{References}

${ }_{380}^{\text {[ [ }}$ [1] D. G. Stamatelos, G. N. Labeas, K. I. Tserpes, Thin-Walled Structures An-

1 alytical calculation of local buckling and post-buckling behavior of isotropic and orthotropic stiffened panels, Thin Walled Structures 49 (3) (2011) 422- 
430. doi:10.1016/j.tws.2010.11.008.

URL http://dx.doi.org/10.1016/j.tws.2010.11.008

[2] R. Degenhardt, A. Kling, H. Klein, W. Hillger, H. C. Goetting, R. Zimmermann, K. Rohwer, Experiments on buckling and postbuckling of thin-walled CFRP structures using advanced measurement systems, International Journal of Structural Stability and Dynamics 7 (2) (2007) 337-358.

[3] A. C. Orifici, R. S. Thomson, Degradation investigation in a postbuckling composite stiffened fuselage panel 82 (2008) 217-224. doi:10.1016/j. compstruct.2007.01.012.

[4] S. Lauterbach, A. C. Orifici, W. Wagner, C. Balzani, H. Abramovich, R. Thomson, Damage sensitivity of axially loaded stringer-stiffened curved CFRP panels, Composites Science and Technology 70 (2) (2010) 240-248. doi:10.1016/j.compscitech.2009.10.013

URL http://dx.doi.org/10.1016/j.compscitech.2009.10.013

[5] R. Zimmermann, H. Klein, A. Kling, Buckling and postbuckling of stringer stiffened fibre composite curved panels Tests and computations, Composite Structures 73 (2006) 150-161. doi:10.1016/j . compstruct.2005.11.050.

${ }_{400}^{4}[6]$ W. Wagner, C. Balzani, Prediction of the postbuckling response of composite airframe panels including ply failure, Engineering Fracture Mechanics 77 (18) (2010) 3648-3657. doi:10.1016/j .engfracmech.2010.05.009.

URL http://dx.doi.org/10.1016/j.engfracmech.2010.05.009

[7] C. D. Mote, Global-local finite element, International Journal for Numerical Methods in Engineering 3 (1971) 565-574.

[8] A. K. Noor, Global-local methodologies and their application to nonlinear analysis, Finite Elements in Analysis and Design 2 (1986) 333-346.

[9] S. C. Mao KM, A Refined Global-Local Finite Element Analysis Method, International Journal for Numerical Methods in Engineering 32 (1991) 2943. 
[10] J. D. Whitcomb, Iterative global/local finite element analysis, Computers and Structures 40 (4) (1991) 1027-1031. doi:10.1016/0045-7949(91) 90334-I.

[11] S. Loehnert, T. Belytschko, A multiscale projection method for macro / microcrack simulations, International Journal for Numerical Methods in Engineering (71) (2007) 1466-1482. doi:10.1002/nme.

[12] G. N. Labeas, S. D. Belesis, I. Diamantakos, K. I. Tserpes, Adaptative Progressive Damage Modeling for Large-scale Composite Structures, International Journal of Damage Mechanics 21(3) (2012) 441-62. doi: $10.1177 / 1056789511400928$

[13] D. Chrupalla, S. Berg, L. Kärger, M. Doreille, T. Ludwig, E. Jansen, R. Rolfes, A. Kling, A homogenization-based two-way multiscale approach for composite structures, In: Rolfes R, Jansen EL, editors. Proceedings of the 3rd ECCOMAS thematic conference on the mechanical response of composites. Germany: Hannover; (2011) p. 263-70.

[14] L. Gendre, O. Allix, P. Gosselet, F. Comte, Non-intrusive and exact global / local techniques for structural problems with local plasticity.

[15] S. Huehne, J. Reinoso, E. Jansen, R. Rolfes, A two-way loose coupling 1. procedure for investigating the buckling and damage behaviour of stiffened ${ }_{430}^{430}$ composite panels, Composite Structures (136) (2016) 513-525. doi:10. 1016/j.compstruct.2015.09.056.

URL http://dx.doi.org/10.1016/j.compstruct.2015.09.056

[16] Abaqus, Writing User Subroutines with ABAQUS (V) (2001) 1-172.

[17] P. Linde, J. Pleitner, H. D. Boer, C. Carmone, Modelling and Simulation of Fibre Metal Laminates, ABAQUS Users' Conference (2004) 421-439.

[18] A. A. Kardak, On an effective submodeling procedure for stresses determined with finite element analysis, Thesis. 
[19] A. E. Bogdanovich, I. Kizhakkethara, Three-dimensional finite element analysis of double-lap composite adhesive bonded joint using submodeling approach, Composites: Part B 30 (1999) 537-551.

[20] C. G. Dávila, P. P. Camanho, Analysis of the effects of residual strains and defects on skin/stiffener debonding using decohesion elements, 44th AIAA/ASME/ASCE/AHS Structures, Structural Dynamics, and Materials Conference. 